\title{
Downregulated miR-204 Promotes Skeletal Muscle Regeneration
}

\author{
Ya Tan $\mathbb{D}^{1,2,3}$ Linyuan Shen $\mathbb{D}^{1,2}$ Mailin Gan $\mathbb{D}^{1,2}$ Yuan Fan $\mathbb{D}^{1,2}$ Xiao Cheng $\mathbb{D}, 1,2$ \\ Ting Zheng $\mathbb{D}^{1,2}$ Lili Niu $\mathbb{D}^{1,2}$, Lei Chen $\mathbb{D}^{1,2}$ Dongmei Jiang $\mathbb{D}^{1,2}$ Xuewei Li $\mathbb{D}^{1,2}$ \\ Shunhua Zhang $\mathbb{D}^{1,2}$ and Li Zhu $\mathbb{D}^{1,2}$ \\ ${ }^{1}$ College of Animal Science and Technology, Sichuan Agricultural University, Chengdu, Sichuan 611130, China \\ ${ }^{2}$ Farm Animal Genetic Resources Exploration and Innovation Key Laboratory of Sichuan Province, Sichuan Agricultural University, \\ Chengdu, Sichuan 611130, China \\ ${ }^{3}$ Institute of Animal Husbandry and Veterinary, Guizhou Academy of Agricultural Science, Guiyang, Guizhou 550005, China
}

Correspondence should be addressed to Shunhua Zhang; 14081@sicau.edu.cn and Li Zhu; zhuli@sicau.edu.cn

Received 22 May 2020; Revised 10 September 2020; Accepted 27 October 2020; Published 17 November 2020

Academic Editor: Aijun Wang

Copyright (C) 2020 Ya Tan et al. This is an open access article distributed under the Creative Commons Attribution License, which permits unrestricted use, distribution, and reproduction in any medium, provided the original work is properly cited.

\begin{abstract}
Skeletal muscle is the most abundant and a highly plastic tissue of the mammals, especially when it comes to regenerate after trauma, but there is limited information about the mechanism of muscle repair and its regeneration. In the present study, we found that miR-204 is downregulated after skeletal muscle injury. In vitro experiments showed that over-expression of miR-204 by transfecting with miR-204 mimics suppressed C2C12 cell proliferation, migration, and blocked subsequent differentiation, whereas inhibition of miR-204 by transfecting with miR-204 inhibitor showed the converse effects. Furthermore, through the dual luciferase reporter system, we demonstrated that miR-204 can target the 3'UTR regions of Pax7, IGF1, and Mef2c and inhibit their expression. Taken together, our results suggest that $P a x 7, I G F 1$, and $M e f 2 c$ are the target genes of miR-204 in the process of myoblasts proliferation, cell migration, and differentiation, respectively, and may contribute to mouse skeletal muscle regeneration. Our results may provide new ideas and references for the skeletal muscle study and may also provide therapeutic strategies of skeletal muscle injury.
\end{abstract}

\section{Introduction}

Tissue and organ regeneration is common in metazoans, and it is puzzling that regenerative capacity has evolved losses in higher animals [1]. Skeletal muscle is one of the mammalian tissues with a strong regenerative capacity [2]. Skeletal muscle represents about $30 \sim 40 \%$ of the total body mass in animals [3]. It is not only a motor organ but also an important energy metabolism and endocrine organ. The ability of the skeletal muscle to regenerate depends on the muscle satellite cells, which are located between the sarcolemma and the basal layer of the skeletal muscle fibers [4]. Usually, the muscle satellite cells remain static, can be activated by being injured, and then change from a static state to a myogenic process. Some of the muscle satellite cells that have proliferated by asymmetric division migrate to the damaged part to differentiate into the damaged part of the myotube repair, and the other part returns to the quiescent state, refilling the satellite cell pool [5].

The activation [6], proliferation [7], migration [8], and differentiation [9] of satellite cells are the main processes of muscle regeneration, and a large number of genes have been found to be involved in the process of skeletal muscle regeneration [10]. C2C12 myoblasts, a cell line that is derived from the satellite cells isolated from murine skeletal muscle, are the best immortalized model to study the reserved myoblast function of satellite cells $[11,12]$. miRNAs are a class of noncoding RNAs of about $22 \mathrm{nt}$ in length that function by inhibiting their target gene expression. With the deepening of miRNA research, some miRNAs have also been found to be involved in the regulation of skeletal muscle regeneration [13]. In recent years, miR-204 has been found to be 
associated with many biological processes, such as cancer [14], inflammation [15], and lipid metabolism [16], but the role of miR-204 in skeletal muscle regeneration is not fully understood.

In this study, we constructed an acute muscle injury model of skeletal muscle in mice, using cardiotoxin (CTX) to detect the expression level of miR-204 during muscle regeneration. Additionally, $\mathrm{C} 2 \mathrm{C} 12$ myoblasts were used as an in vitro model to investigate the function of miR-204 in the proliferation, migration, and differentiation of $\mathrm{C} 2 \mathrm{C} 12$ myoblasts by overexpression or inhibition of miR-204. All the results of this study may be able to elucidate the effects of miR-204 on skeletal muscle regeneration and provide valuable information for the diagnosis and treatment of muscle damage.

\section{Materials and Methods}

2.1. Animals and Treatment. Animals were housed in the Animal Resources under controlled temperature $\left(22^{\circ} \mathrm{C} \pm 3^{\circ} \mathrm{C}\right)$ for a natural light cycle and allowed ad libitum to feed and water. ICR mice ( $\$, 8$ weeks) were purchased from the Chengdu Da Shuo Experimental Animal Co., Ltd. (Chengdu, China). A model of anterior tibial muscle (TA) injury was induced by injection of cytotoxin (CTX, Sigma, USA) with the dosage of $10 \mu \mathrm{M}$ [17]. All the experimental procedures and sample collection were approved by the Institutional Animal Care and Use Committee of the College of Animal Science and Technology of Sichuan Agricultural University, Sichuan, China, under permit No. DKY2018102010.

2.2. Tissue Section. The obtained mouse TA was fixated with $4 \%$ paraformaldehyde; then, the tissues were dehydrated using ethanol, infiltrated, and embedded using paraffin; and finally, the tissue sections (4-5 $\mu \mathrm{M}$ thickness) were stained with hematoxylin and eosin $(\mathrm{H} \& \mathrm{E})$ for histological analysis.

2.3. Cell Culture and Treatment. C2C12 myoblasts purchased from Stem Cell Bank, Chinese Academy of Science, were cultured at $37^{\circ} \mathrm{C}$ in a $\mathrm{CO}_{2}$ incubator $\left(5 \% \mathrm{CO}_{2}\right)$. The $\mathrm{C} 2 \mathrm{C} 12$ cells were maintained in Dulbecco's modified Eagle's medium (DMEM, Gibco, Carlsbad, CA, USA) and supplemented with $10 \%$ fetal bovine serum. When the $\mathrm{C} 2 \mathrm{C} 12$ myoblasts reached $80 \%$ confluence, the cells were moved to differentiation medium (DM) which consist of DMEM and 2\% horse serum (Gibco). miR-204 mimic, inhibitor, and negative control (Ribobio, Guangzhou, China) were transfected into the C2C12 myoblasts using Lipofectamine 3000 (Invitrogen, USA) [18].

2.4. Cell Proliferation Assay. Cell proliferation assays refer to previous studies [19]. The CCK-8 kit (Beyotime, Shanghai, China) was used at 24 hours, 48 hours, and 96 hours after transfection according to the manufacturer's protocol. Forty-eight hours after transfection, $10 \mu \mathrm{M}$ EdU (Ribobio, Guizhou, China) was added to the growth medium, and the cells were cultured for $2 \mathrm{~h}$. Subsequently, the EdU staining was performed according to the manufacturer's protocol.
2.5. Scratch Test. The C2C12 myoblasts were manually scraped off with a p200 pipette tip to create scratches $[20,21]$. After the scratch defect was made, the cells were washed in PBS and cultured in DMEM. The C2C12 myoblasts were then incubated at $37^{\circ} \mathrm{C}$ for 0,12 , or 24 hours; observed; and photographed.

2.6. Immunocytochemistry. The differentiated C2C12 myoblasts were washed with PBS, fixed with $4 \%$ paraformaldehyde for 20 minutes, and permeabilized with $0.2 \%$ Triton $\mathrm{X}-100$ for 10 minutes at room temperature $\left(22^{\circ} \mathrm{C} \pm 3^{\circ} \mathrm{C}\right)$. Then, nonspecific antibody binding was blocked with $5 \%$ goat serum (Bioss, Beijing, China) for $2 \mathrm{~h}$ and subsequently incubated with anti-MyHC (ab51263, Abcam, USA) overnight at $4^{\circ} \mathrm{C}$. The next day, the $\mathrm{C} 2 \mathrm{C} 12$ myoblasts were incubated with Alexa Fluor ${ }^{\circledR} 488$ secondary antibody (ab150113; Abcam) for 1h. Finally, the cells were observed on a fluorescence microscope (Olympus, Kyoto, Japan) [22].

2.7. Luciferase Reporter Assay. The 3'UTR of Pax7 (Paired box 7), IGF1 (insulin-like growth factor 1), and Mef2c (myocyte enhancer factor2) (TsingKe Biotech, Chengdu, China) containing the miR-204 binding site was inserted into the psiCHECKTM-2 vector (Promega, USA), and then, the psiCHECKTM-2 vector and miR-204 mimic were cotransfected into HeLa cells (ATCC, USA) using the Lipofectamine $^{\mathrm{TM}} 3000$ reagent (Invitrogen, USA). Finally, the Firefly fluorescence and Renilla fluorescence were measured using the Dual-Glo Luciferase Assay System (Promega, Madison, USA) [23].

2.8. Quantitative Real-Time PCR ( $q R T-P C R)$. qRT-PCR was performed using the $\mathrm{SYBR}^{\circledR}$ Premix Ex Taq ${ }^{\mathrm{TM}}$ kit (TaKaRa) on a CFX96 Real-Time PCR detection system (Bio-Rad, Richmond, CA, USA). The relative expression of mRNAs and miRNAs was calculated using the $2^{-\Delta \Delta \mathrm{Ct}}$ method [24]. The sequences of primers for qRT-PCR are listed in Table 1.

2.9. Statistical Analyses. All quantitative results are presented as mean \pm SD. Statistical analyses were conducted using the SPSS 20.0 software. Unpaired Student's $t$-test was performed for comparison between the treated and untreated control (NC) groups, and one-way ANOVA analysis was conducted for comparing multiple groups followed by a Dunnett's method post hoc analysis. Statistical significance was defined as $p<0.05$.

\section{Results}

3.1. Downregulation of miR-204 Expression after Muscle Injury. After CTX (cardiotoxin) injection, a mouse TA (tibialis anterior) muscle injury model was successfully constructed. The HE staining of the TA muscle showed that a large number of muscle fibers were dissolved after CTX injection for $6 \mathrm{~h}$, and inflammatory infiltration occurred after $1 \mathrm{~d}$. A large number of macrophages appeared at 3 days, and new muscle fibers were formed at 7 days. On $21 \mathrm{~d}$, the muscle fibers were basically repaired, but there were still a certain number of central nuclear muscle fibers (Figure 1(a)). In the process of muscle regeneration, the expression level of 
TABLE 1: The primers used for qRT-PCR and the synthesized RNA oligonucleotides.

\begin{tabular}{|c|c|c|}
\hline Gene & Primer sequence $\left(5^{\prime}-3^{\prime}\right)$ & $\mathrm{TM} /{ }^{\circ} \mathrm{C}$ \\
\hline \multirow{2}{*}{ Pax3 } & F-CCGGGGCAGAATTACCCAC & \multirow{2}{*}{60.6} \\
\hline & R-GCCGTTGATAAATACTCCTCCG & \\
\hline \multirow{2}{*}{ Pax7 } & F-TGGGGTCTTCATCAACGGTC & \multirow{2}{*}{60} \\
\hline & R-ATCGGCACAGAATCTTGGAGA & \\
\hline \multirow{2}{*}{ IGF1 } & F-GGACGGGGACTTCTGAGTCTT & \multirow{2}{*}{60.6} \\
\hline & R-AGAGAGCGGGACTCCTTCTG & \\
\hline \multirow{2}{*}{$\operatorname{Mef} 2 c$} & F-ATCTCTCСCTGCCTTCTACTC & \multirow{2}{*}{60} \\
\hline & R-CTCCCATCGTAGGAACTGCT & \\
\hline \multirow{2}{*}{ MyoG } & F-GCCCAGTGAATGCAACTCCCACA & \multirow{2}{*}{58} \\
\hline & R-CAGCCGCGAGCAAATGATCTCCT & \\
\hline \multirow{2}{*}{ MyoD } & F-AGACTTCTATGATGACCCGTGTT & \multirow{2}{*}{57} \\
\hline & R-TCAGCGTTGGTGGTCTTGC & \\
\hline \multirow{2}{*}{$\mathrm{MyHC}$} & F-GCGAATCGAGGCTCAGAACAA & \multirow{2}{*}{60} \\
\hline & R-GTAGTTCCGCCTTCGGTCTTG & \\
\hline \multirow{2}{*}{$C C N B$} & F-AAGGTGCCTGTGTGTGAACC & \multirow{2}{*}{61} \\
\hline & R-GTCAGCCCCATCATCTGCG & \\
\hline \multirow{2}{*}{$C C N D$} & F-GCGTACCCTGACACCAATCTC & \multirow{2}{*}{61} \\
\hline & R-CTCCTCTTCGCACTTCTGCTC & \\
\hline \multirow{2}{*}{ CCNE } & F-GTGGCTCCGACCTTTCAGTC & \multirow{2}{*}{60} \\
\hline & R-CACAGTCTTGTCAATCTTGGCA & \\
\hline \multirow{2}{*}{ CDK4 } & F-ATGGCTGCCACTCGATATGAA & \multirow{2}{*}{60} \\
\hline & R-TCCTCCATTAGGAACTCTCACAC & \\
\hline \multirow{2}{*}{$\beta$-Actin } & F-TGGAATCCTGTGGCATC CATGAAAC & \multirow{2}{*}{60} \\
\hline & R-TAAAACGCAGCTCAG TAACAGTCCG & \\
\hline \multirow{2}{*}{$m i R-204$} & F-TTCCCTTTGTCATCCTATGCCT & \multirow{2}{*}{60} \\
\hline & R- Uni-miR qPCR primer, included in kit (miRNA universal downstream primer, TaKaRa) & \\
\hline \multirow{2}{*}{ U6 } & F: CTCGCTTCGGCAGCACA & \multirow{2}{*}{60} \\
\hline & R: AACGCTTCACGAATTTGCGT & \\
\hline
\end{tabular}

F: forward; R: reverse.

Pax7, a marker of muscle satellite cells, showed a pattern of increasing first and then decreasing (Figure 1(b)). Compared with the control group, the expression level of miR-204 showed a trend of decreasing first and then increasing and maintained a lower state throughout the muscle regeneration process (Figure 1(c)).

Pax7 Is a Target Gene of miR-204 That Regulates the Proliferation of C2C12 Myoblasts

To further investigate the function of miR-204 during skeletal muscle regeneration, the TargetScan software and sequence alignment analysis were applied in the miR-204 target finding. Then, we found miR-204 binding sites in the 3'UTR region of Pax7 (Figure 2(a)). Subsequently, we used the dual-luciferase reporter system and found that the overexpression of miR-204 significantly inhibited the luciferase activity of the wild type of $\operatorname{Pax} 7$ (Figure 2(b)). As shown in Figure 2(c), compared with the control group, the expression of miR-204 in the mimic group was elevated by approximately 5-fold. However, the expression was down to $31 \%$ in the inhibitor group. Compared with the negative control group, the expression of Pax7, CCNB, CCND, CCNE, and
CDK4 in C2C12 myoblasts was significantly downregulated by the miR-204 mimic. Meanwhile, the miR-204 inhibitor significantly promoted the expression of these genes (Figures 2(c) and 2(e)). Furthermore, the CCK8 results confirmed that miR-204 suppressed C2C12 myoblast proliferation (Figure 2(f)). In addition, these results were further verified by the EdU assay, that miR-204 mimic decreased the ratio of EdU-positive cells (Figures 2(g) and 2(h)). All these results suggest that miR-204 can inhibit C2C12 myoblast proliferation probably by targeting $\operatorname{Pax} 7$.

3.2. IGF1 Is a Target Gene of miR-204 That Regulates the Migration of C2C12 Myoblasts. The migration of satellite cells to the injury site is a key process in the regeneration of skeletal muscles [25]. IGF1 was previously reported to involve in cell migration, as well as in skeletal muscle hypertrophy [26, 27]. Meanwhile, miR-204 has also been found to inhibit the migration of osteosarcoma [28]. Taken together, it is possible; therefore, we hypothesized that IGF1 and miR204 are related to the myocyte migration. Interestingly, we found that the 3'UTR region of the IGF1 has binding sites 


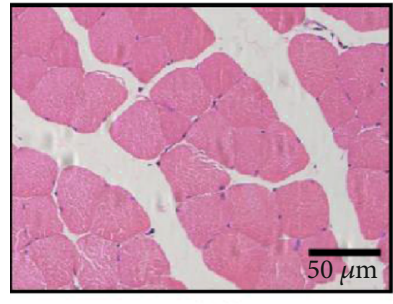

NC

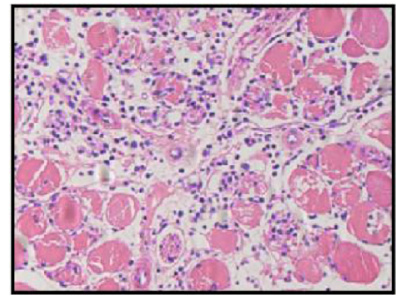

$3 \mathrm{~d}$

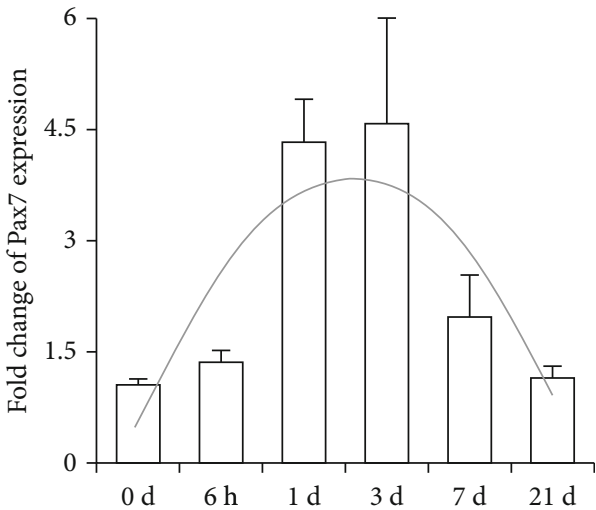

(b)

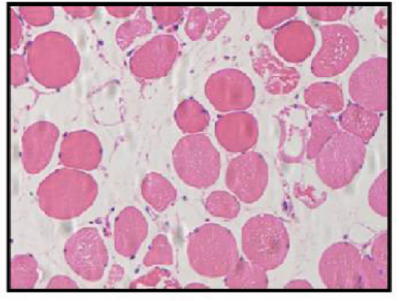

$6 \mathrm{~h}$

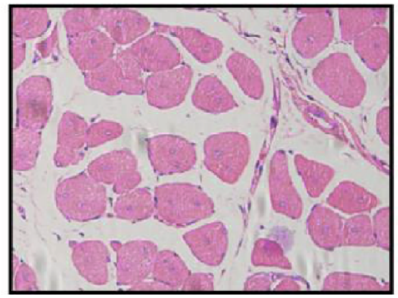

$7 \mathrm{~d}$

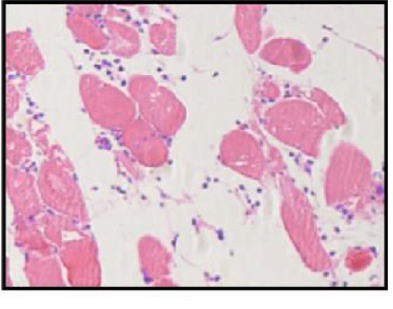

$1 \mathrm{~d}$

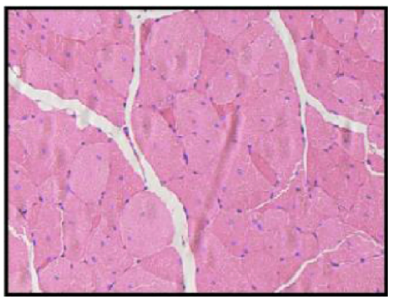

$21 \mathrm{~d}$

(a)

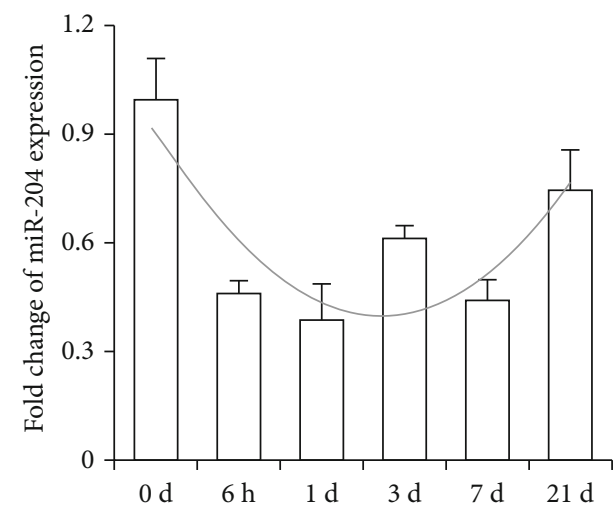

(c)

FIGURE 1: Downregulation of miR-204 expression after muscle injury. (a) HE staining of mouse TA muscle. Scale bar $=50 \mu \mathrm{m}, n=8$. (b), (c) The expression of $\operatorname{Pax} 7$ (Paired box 7) and miR-204 in the TA muscle of mice after injury. $n=3$. Data are presented as means \pm SD.

for miR-204 (Figure 3(a)). Further, we confirmed the binding of miR-204 to IGF1 by a dual-luciferase reporter system (Figure 3(b)). The miR-204 mimic significantly inhibited the expression of IGF1 and Pax3, which is the muscle satellite cell migration marker gene (Figure 3(c)). In addition, the scratch test showed that the miR-204 mimic significantly inhibited cell migration, while the miR-204 inhibitor significantly promoted cell migration (Figures 3(d) and 3(e)). All these results suggest that miR-204 may inhibit the migration of C2C12 myoblasts through IGF1.

\subsection{Mef2c Is a Target Gene of miR-204 That Regulates the} Differentiation of C2C12 Myoblasts. Further, we found and confirmed the binding relationship between miR-204 and $M e f 2 c$ (Figures 4(a) and 4(b)), which was reported to promote myoblasts differentiation. Compared with the negative control group, the expression level of miR-204 in the mimic group was increased by approximately 21 -fold in C2C12 myotubes. However, the expression of miR-204 was down to $45 \%$ in the inhibitor group (Figure $4(\mathrm{c})$ ).
As shown in Figure 4(d), compared with the control group, the expression of $M y o G, M e f 2 c$, and $M y H C$ in C2C12 myoblasts was significantly downregulated by the miR-204 mimic. On the contrary, the expression of $M y o D$, $M e f 2 c$, and $M y H C$ was significantly increased after the miR-204 inhibitor transfection (Figure $4(\mathrm{~d})$ ). $M y H C$ is a myogenic differentiation marker of myocytes, which was stained by MyHC antibody. The immunocytochemistry results showed that myocytes fusion rate was significantly decreased after the transfection of miR-204 mimic, whereas the fusion index was increased by transfection of miR-204 inhibitor (Figures 4(e) and 4(f)).

\section{Discussion}

In the present study, we found that miR-204 is downregulated during skeletal muscle regeneration in vivo. The dualluciferase reporter system and qRT-PCR demonstrated that miR-204 can bind to the 3'UTR of Pax7, IGF1, and Mef2c and then inhibit their expression level. Cell proliferation assay, scratch assay, and differentiation assay showed that 


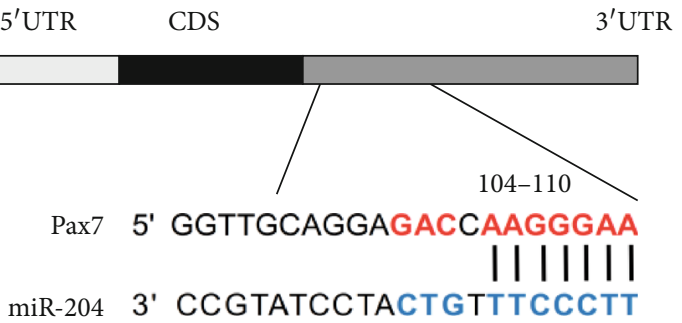

(a)

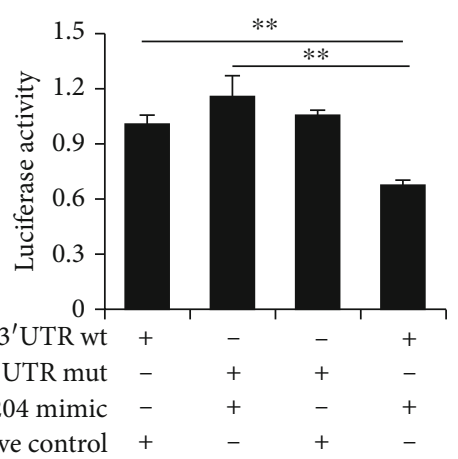

(b)

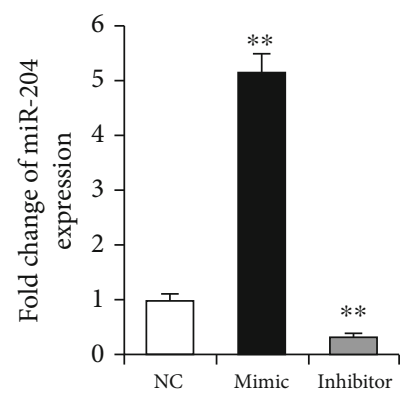

(c)

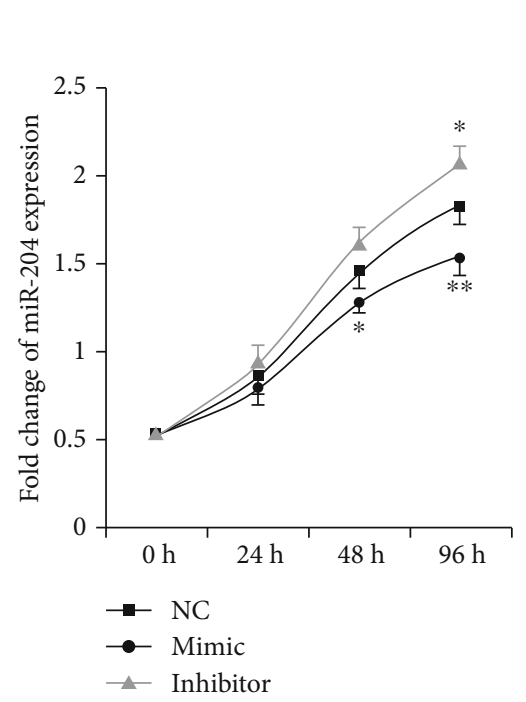

(f)

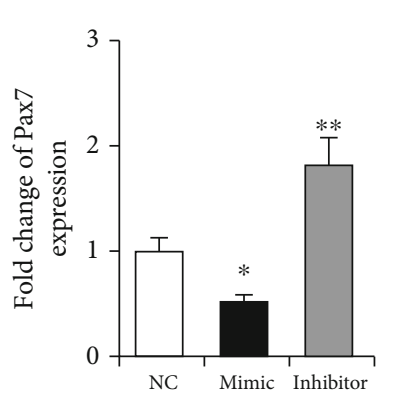

(d)

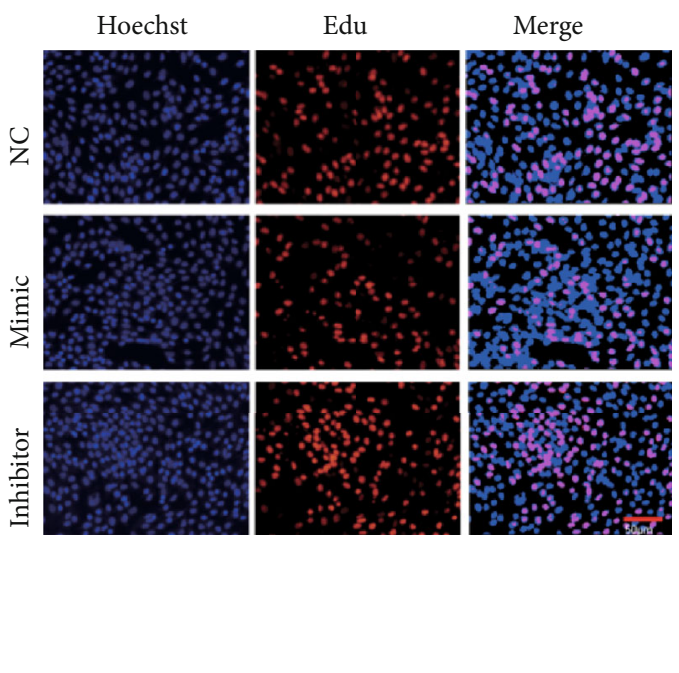

(g)

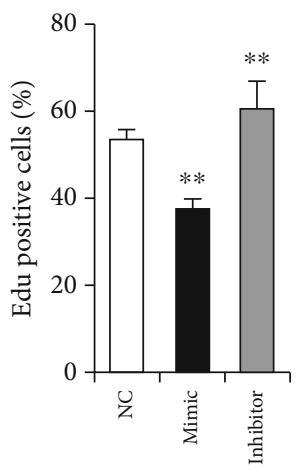

(h)

FIgURe 2: miR-204 regulates C2C12 myoblast proliferation through Pax7. (a) The binding site of miR-204 to Pax7. (b) The Pax7 3'UTR with wild-type (wt) or mutant type (mut) were inserted into psiCHECK ${ }^{\mathrm{TM}}-2$ vectors and cotransfected with miR-204 mimic or negative control into HeLa cells, respectively. $n=3$. (c-e) The expression of miR-204 (c), Pax7 (d), and cell division cycle genes (CCNB (Cyclin B), CCND (Cyclin D), CCNE (Cyclin E), CDK4 (Cyclin-dependent kinase 4)) in the C2C12 myoblasts after transfection with miR-204 mimic or inhibitor. $n=3$. (f) Cell counts measured using the Cell Count Kit 8 (CCK-8) method. $n=6$. (g, h) The EdU assay was carried out after a 48h transfection. C2C12 myoblasts undergoing DNA replication were stained by EdU (red), and cell nuclei were stained with Hoechst (blue). Scale bar $=50$ $\mu \mathrm{m}, n=3$. Data are presented as means $\pm \mathrm{SD} .{ }^{*} p<0.05,{ }^{* *} p<0.01$.

miR-204 can inhibit the proliferation, migration, and differentiation through its corresponding targets in C2C12 myoblasts, respectively (Figure 5).
Pax7 is exclusively expressed in satellite cells and plays a crucial role in self-renewal and myogenic potential maintenance in adult skeletal muscle [29]. In the present 


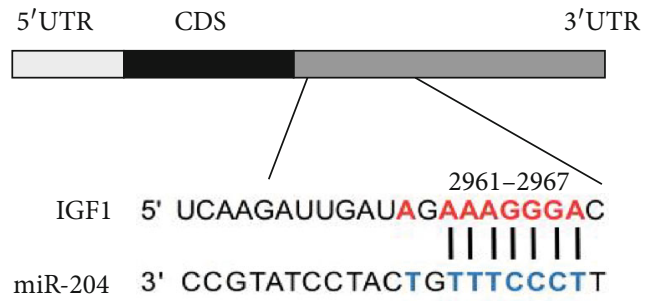

(a)

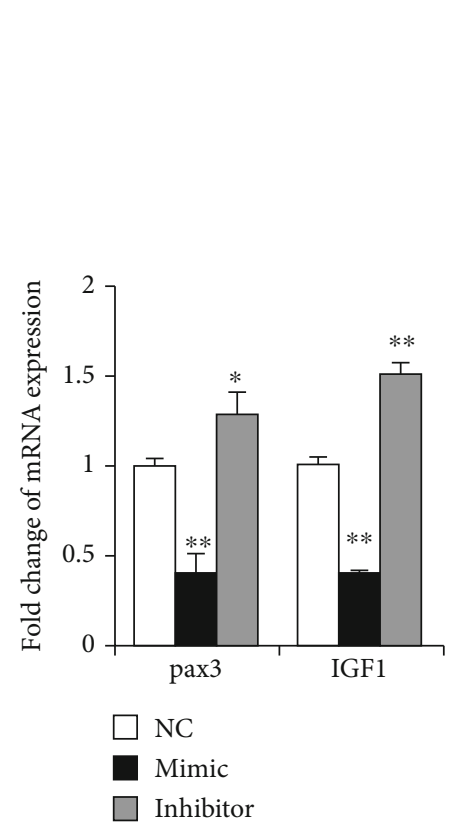

(c)
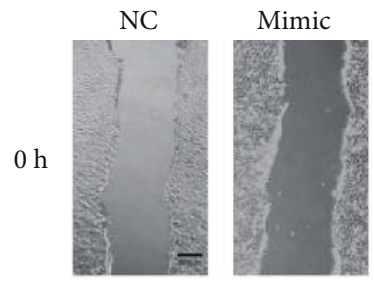

$24 \mathrm{~h}$
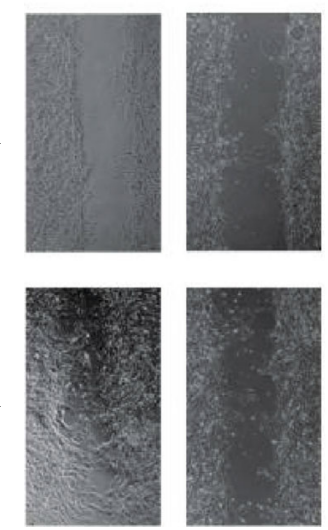

(d)

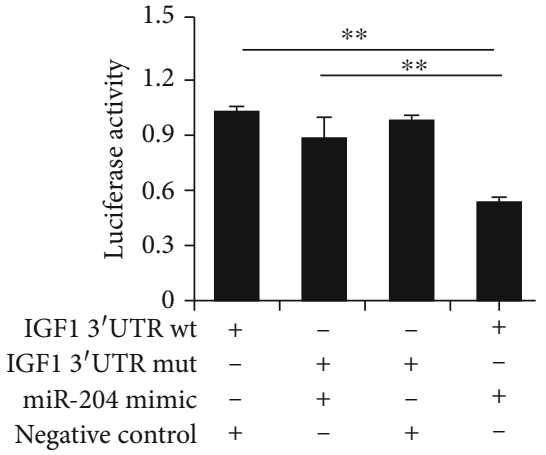

(b)
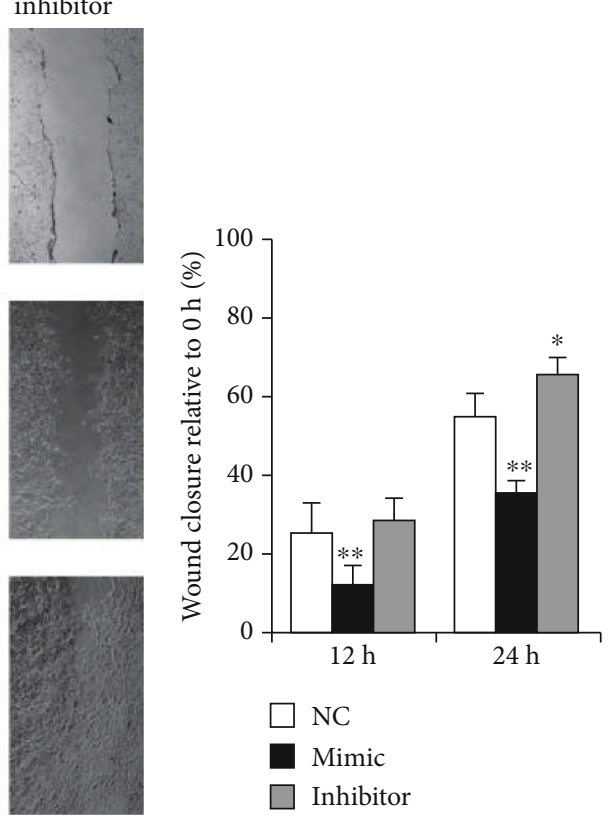

(e)

FIGURE 3: miR-204 regulates C2C12 myoblast migration through IGF1 (insulin-like growth factor 1). (a) The binding site of miR-204 to IGF1. (b) The IGF1 3'UTR with wild-type (wt) or mutant type (mut) were inserted into the psiCHECK ${ }^{\mathrm{TM}}-2$ vectors and cotransfected with miR-204 mimic or negative control into HeLa cells, respectively. $n=3$. (c) The expression of Pax3 (Paired box 3 ) and IGF1 in the C2C12 myoblasts after transfection with miR-204 mimic or inhibitor. $n=3$. (d, e) Scratch test pictures of cell monolayers at time 0 , 12 hours, and 24 hours following initiation of scratch defect on C2C12 myoblasts. Scale bar $=200 \mu \mathrm{m}, n=3$. Data are presented as means \pm SD. ${ }^{*} p<0.05,{ }^{* *} p<0.01$.

study, it was found that the expression level of $\operatorname{Pax} 7$ increased significantly on the 1st day after muscle injury, reached the highest level at $3 \mathrm{~d}$, and began to fall back after 7d. miR-204 exhibits an opposite expression pattern to Pax7. All these results suggest that miR-204 may also play a crucial role in skeletal muscle regeneration. Furthermore, the dual-luciferase reporter system confirmed the binding relationship between miR-204 and Pax7. In previous researches, miR-204 has been reported to have the ability to inhibit the proliferation of a variety of normal cells [30] and cancer cells [31]. In our study, we consistently found that miR-204 can inhibit the expression of $C C N B$, $C C N D, C C N E$, and $C D K 4$, which promote cell cycle progression [32, 33]. In addition, CCK8 and EdU assays showed that miR-204 mimic significantly inhibited cell proliferation while the miR-204 inhibitor significantly promoted cell proliferation.

Pax3 and IGF1 were found to be involved in cell migration [34, 35]. miR-204 has also been found to inhibit the migration of osteosarcoma [28]. Interestingly, we found and confirmed the binding relationship between miR-204 and IGF1. In this study, cell scratch assays also showed that miR-204 can inhibit the migration of $\mathrm{C} 2 \mathrm{C} 12$ myoblasts. These results suggest that the role of miR-204 in inhibiting cell migration may exist among various types of cells.

When the satellite cells complete proliferation and migrate to the muscle injury site, the myocytes begin to 


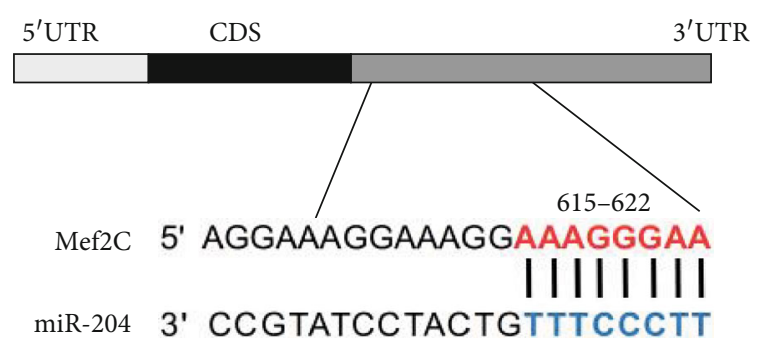

(a)

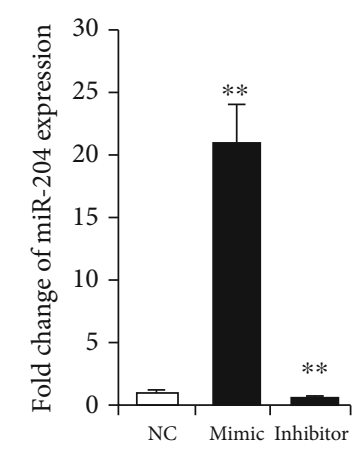

NC

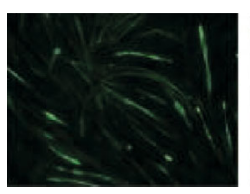

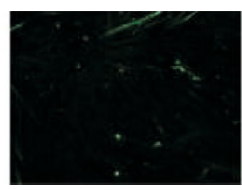

Mimic

(e)

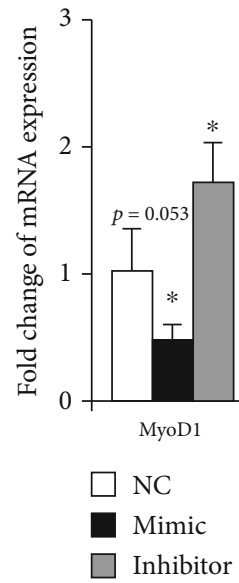

Inhibitor

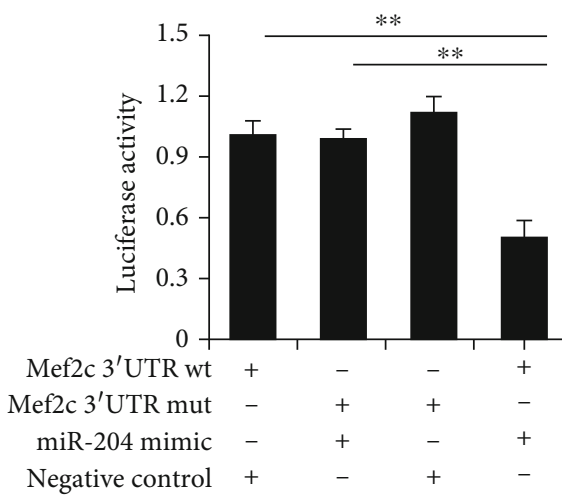

(b) (c)

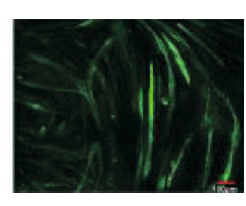

Inhibitor (d)

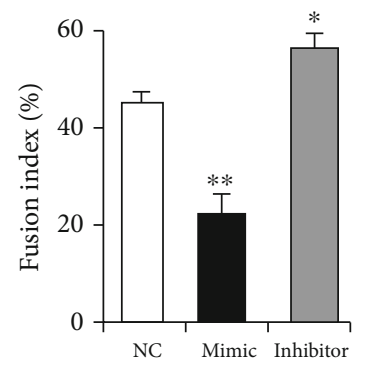

(f)

FIGURE 4: miR-204 regulates C2C12 myoblasts differentiation through Mef2c (myocyte enhancer factor 2c). (a) The binding sites of miR-204 to $M e f 2 c$. (b) The Mef2c 3'UTR with wild-type (wt) or mutant type (mut) were inserted into the psiCHECK ${ }^{\mathrm{TM}}-2$ vectors and cotransfected with miR-204 mimic or negative control into HeLa cells, respectively. $n=3$. (c, d) The expression of miR-204, MyoD, MyoG, Mef2c, and MyHC in the $\mathrm{C} 2 \mathrm{C} 12$ myotubes after transfection with miR-204 mimic or inhibitor. $n=3$. (e, f) Immunofluorescence of MyHC (muscle myosin heavy chain) in C2C12 myotubes. Scale bar $=100 \mu \mathrm{m}, n=3$. Data are presented as means \pm SD. ${ }^{*} p<0.05,{ }^{* *} p<0.01$.

differentiate and fuse with each other to form multinucleated myotubes $[2,36]$. MRFs (myogenic regulatory factors) and Mef2c (myocyte enhancer factor 2c) have the ability to promote satellite cell differentiation, which were essential during the myogenesis process [22, 27]. During the differentiation of C2C12 myoblasts, miR-204 inhibited its target gene $M e f 2 c$ and also decreased the expression level of $M y o D, M y o G$, and $M y H C$. And further immunofluorescence staining verified that miR-204 inhibited the myocyte differentiation through $M e f 2 c$.

\section{Conclusion}

In summary, we focused on the expression and function of miR-204 during skeletal muscle regeneration in the present study. The results suggest that the downregulation of miR204 expression may be associated with skeletal muscle regeneration. Furthermore, our results show that inhibition of miR-204 promoted the expression of Pax7, IGF1, and Mef $2 c$, which are the target genes of miR-204, and are also closely related to skeletal muscle regeneration. These results suggest 


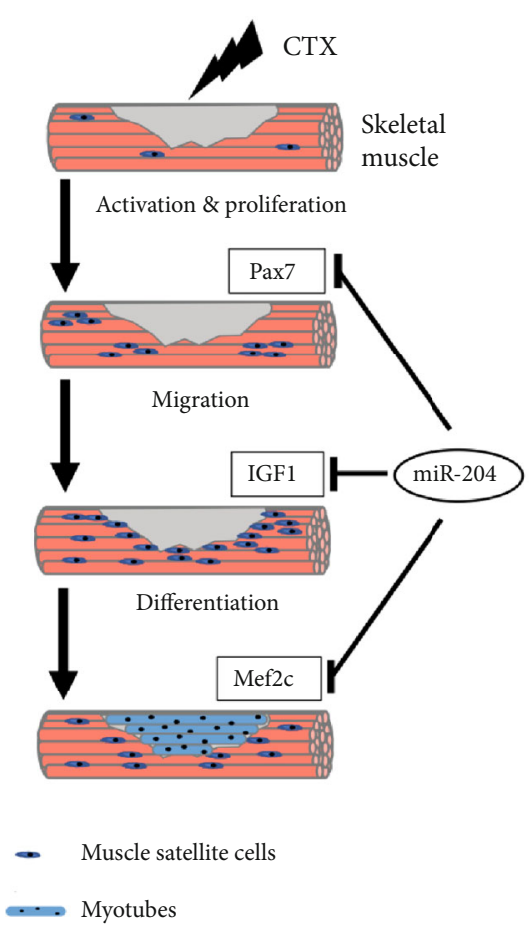

FIGURE 5: Schematic showing the regulation pattern of miR-204 on skeletal muscle. miR-204 can target Pax7, IGF1, and Mef $2 c$ in the process of myoblast proliferation, cell migration, and differentiation, respectively, and may contribute to the CTXmediated mouse skeletal muscle injury.

that miR-204 may regulate the activation, proliferation, migration, and differentiation of myocytes through Pax7, $I G F 1$, and Mef2c. Our findings reveal the potential of miR204 in the treatment of skeletal muscle-related diseases.

\section{Data Availability}

The data used to support the findings of this study are included in the article.

\section{Conflicts of Interest}

The authors declare that they have no conflicts of interest.

\section{Authors' Contributions}

Ya Tan and Linyuan Shen contributed equally to this work.

\section{Acknowledgments}

This study was supported by the National Key Research and Development Program of China (No. 2018YFD0501004), the National Natural Science Foundation of China (No. 31530073), the Sichuan Science and Technology Support Program (No. 2016NYZ0050; No. SCSZTD-3-008), the Earmarked Fund for China Agriculture Research System (No. CARS-36-05B), and Guizhou Science and Technology Department, China (No. QKHZC20182280; No. QKHZC20192278).

\section{References}

[1] X. H. Parsons, Y. D. Teng, D. A. Moore, and E. Y. Snyder, "Patents on technologies of human tissue and organ regeneration from pluripotent human embryonic stem cells," Recent patents on regenerative medicine, vol. 1, no. 2, pp. 142-163, 2011.

[2] M. B. Baghdadi and S. Tajbakhsh, "Regulation and phylogeny of skeletal muscle regeneration," Developmental Biology, vol. 433 , no. 2, pp. 200-209, 2018.

[3] L. Shen, J. Du, Y. Xia et al., "Genome-wide landscape of DNA methylomes and their relationship with mRNA and miRNA transcriptomes in oxidative and glycolytic skeletal muscles," Scientific Reports, vol. 6, no. 1, 2016.

[4] B. C. Collins, R. W. Arpke, A. A. Larson et al., "Estrogen regulates the satellite cell compartment in females," Cell Reports, vol. 28, no. 2, pp. 368-381.e6, 2019.

[5] G. Xiong, S. M. Hindi, A. K. Mann et al., "The PERK arm of the unfolded protein response regulates satellite cell-mediated skeletal muscle regeneration," eLife, vol. 6, article e22871, 2017.

[6] G. J. Schaaf, T. J. van Gestel, E. Brusse et al., "Lack of robust satellite cell activation and muscle regeneration during the progression of Pompe disease," Acta Neuropathologica Communications, vol. 3, no. 1, p. 65, 2015.

[7] L. Zhu, L. Hou, J. Ou et al., "MiR-199b represses porcine muscle satellite cells proliferation by targeting JAG1," Gene, vol. 691, pp. 24-33, 2019.

[8] K. Kowalski, A. Kołodziejczyk, M. Sikorska et al., "Stem cells migration during skeletal muscle regeneration-the role of Sdf-1/Cxcr4 and Sdf-1/Cxcr7 axis," Cell Adhesion \& Migration, vol. 11, no. 4, pp. 384-398, 2017.

[9] W. Zhang, Y. Xu, L. Zhang et al., "Synergistic effects of TGF $\beta 2$, WNT9a, and FGFR4 signals attenuate satellite cell differentiation during skeletal muscle development," Aging Cell, vol. 17, no. 4, article e12788, 2018.

[10] T. Chaillou1 and J. T. Lanner, "Regulation of myogenesis and skeletal muscle regeneration: effects of oxygen levels on satellite cell activity," The FASEB Journal, vol. 30, no. 12, pp. 3929-3941, 2016.

[11] P. Senesi, L. Luzi, A. Montesano, N. Mazzocchi, and I. Terruzzi, "Betaine supplement enhances skeletal muscle differentiation in murine myoblasts via IGF-1 signaling activation," Journal of Translational Medicine, vol. 11, no. 1, p. 174, 2013.

[12] M. Kurosaka, Y. Ogura, T. Funabashi, and T. Akema, "Early growth response 3 (Egr3) contributes a maintenance of C2C12 myoblast proliferation," Journal of Cellular Physiology, vol. 232, no. 5, pp. 1114-1122, 2017.

[13] J. Wang, L. Z. Yang, J. S. Zhang et al., "Effects of microRNAs on skeletal muscle development," Gene, vol. 668, pp. 107113, 2018.

[14] T. Li, H. Pan, and R. Li, "The dual regulatory role of miR-204 in cancer," Tumor Biology, vol. 37, no. 9, pp. 11667-11677, 2016.

[15] H. Li, J. Wang, X. Liu, and Q. Cheng, "MicroRNA-204-5p suppresses IL6-mediated inflammatory response and chemokine generation in HK-2 renal tubular epithelial cells by targeting IL6R," Biochemistry and Cell Biology, vol. 97, no. 2, pp. 109$117,2019$.

[16] M. Civelek, R. Hagopian, C. Pan et al., "Genetic regulation of human adipose microRNA expression and its consequences 
for metabolic traits," Human Molecular Genetics, vol. 22, no. 15, pp. 3023-3037, 2013.

[17] M. A. A. Mahdy, H. Y. Lei, J.-I. Wakamatsu, Y. Z. Hosaka, and T. Nishimura, "Comparative study of muscle regeneration following cardiotoxin and glycerol injury," Annals of AnatomyAnatomischer Anzeiger, vol. 202, pp. 18-27, 2015.

[18] M. Gan, J. Du, L. Shen et al., "miR-152 regulates the proliferation and differentiation of $\mathrm{C} 2 \mathrm{C} 12$ myoblasts by targeting E2F3," In Vitro Cellular \& Developmental Biology-Animal, vol. 54, no. 4, pp. 304-310, 2018.

[19] M. Gan, D. Yang, Y. Fan et al., "Bidirectional regulation of genistein on the proliferation and differentiation of $\mathrm{C} 2 \mathrm{C} 12$ myoblasts," Xenobiotica, vol. 50, no. 11, pp. 1352-1358, 2020.

[20] J. X. Liu, J. A. Werner, J. A. Buza, T. Kirsch, J. D. Zuckerman, and M. S. Virk, "Povidone-iodine solutions inhibit cell migration and survival of osteoblasts, fibroblasts, and myoblasts," Spine, vol. 42, no. 23, pp. 1757-1762, 2017.

[21] E. S. Yahaya, W. Cordier, P. A. Steenkamp, and V. Steenkamp, "Attenuation of oxidative stress and artificial wound closure in C2C12 myoblasts induced by sequential extracts of Boerhavia diffusa," Journal of Pharmacy and Pharmacology, vol. 70, no. 1, pp. 111-123, 2018.

[22] X. Cheng, J. Du, L. Shen et al., "MiR-204-5p regulates C2C12 myoblast differentiation by targeting MEF2C and ERR $\gamma$," Biomedicine \& Pharmacotherapy, vol. 101, article S0753332217370403, pp. 528-535, 2018.

[23] L. Shen, M. Gan, Z. Tan et al., "A novel class of tRNA-derived small non-coding RNAs respond to myocardial hypertrophy and contribute to intergenerational inheritance," Biomolecules, vol. 8, no. 3, p. 54, 2018.

[24] K. J. Livak and T. D. Schmittgen, "Analysis of relative gene expression data using real-time quantitative PCR and the 2$\Delta \Delta$ CT method," Methods, vol. 25no. 4, pp. 402-408, 2001.

[25] L. Xiao and K. K. H. Lee, "BRE facilitates skeletal muscle regeneration by promoting satellite cell motility and differentiation," Biology Open, vol. 5, no. 2, pp. 100-111, 2016.

[26] C. E. Perrone, D. Fenwick-Smith, and H. H. Vandenburgh, "Collagen and stretch modulate autocrine secretion of insulin-like growth factor-1 and insulin-like growth factor binding proteins from differentiated skeletal muscle cells," The Journal of Biological Chemistry, vol. 270, no. 5, pp. 2099-2106, 1995.

[27] N. Zanou and P. Gailly, "Skeletal muscle hypertrophy and regeneration: interplay between the myogenic regulatory factors (MRFs) and insulin-like growth factors (IGFs) pathways," Cellular and Molecular Life Sciences, vol. 70, no. 21, pp. 41174130, 2013.

[28] M. Li, Y. Shen, Q. Wang, and X. Zhou, "MiR-204-5p promotes apoptosis and inhibits migration of osteosarcoma via targeting EBF2," Biochimie, vol. 158, pp. 224-232, 2019.

[29] Y. Chen, G. Lin, and J. M. Slack, "Control of muscle regeneration in the Xenopus tadpole tail byPax7," Development, vol. 133, no. 12, pp. 2303-2313, 2006.

[30] Y. Jin, J. Wang, M. Zhang et al., "Role of bta-miR-204 in the regulation of adipocyte proliferation, differentiation, and apoptosis," Journal of cellular physiology, vol. 234, no. 7, pp. 11037-11046, 2018.

[31] Y. Xia, Y. Zhu, T. Ma et al., "miR-204 functions as a tumor suppressor by regulating SIX1 in NSCLC," FEBS letters, vol. 588, no. 20, pp. 3703-3712, 2014.
[32] T. Yoshihara, D. Collado, and M. Hamaguchi, "Cyclin D1 down-regulation is essential for DBC2's tumor suppressor function," Biochemical and Biophysical Research Communications, vol. 358, no. 4, pp. 1076-1079, 2007.

[33] Y. Takano, Y. Kato, P. J. van Diest, M. Masuda, H. Mitomi, and I. Okayasu, "Cyclin D2 overexpression and lack of p27 correlate positively and cyclin E inversely with a poor prognosis in gastric cancer cases," The American Journal of Pathology, vol. 156, no. 2, pp. 585-594, 2000.

[34] M. Buckingham and F. Relaix, "PAX3 and PAX7 as upstream regulators of myogenesis," in Seminars in cell \& developmental biology, vol. 44, pp. 115-125, Elsevier, 2015.

[35] Z. Li, R. Jiang, Q. Yue, and H. Peng, "MicroRNA-29 regulates myocardial microvascular endothelial cells proliferation and migration in association with IGF1 in type 2 diabetes," Biochemical and Biophysical Research Communications, vol. 487, no. 1, pp. 15-21, 2017.

[36] M. Schmidt, S. C. Schüler, S. S. Hüttner, B. von Eyss, and J. von Maltzahn, "Adult stem cells at work: regenerating skeletal muscle," Cellular and Molecular Life Sciences, vol. 76, no. 13, pp. 2559-2570, 2019. 